\title{
Circulating adiponectin levels and risk of endometrial cancer: Systematic review and meta-analysis
}

\author{
ZHI-JUN LI ${ }^{1 *}$, XUE-LING YANG ${ }^{2 *}$, YAN YAO ${ }^{1}$, WEI-QING HAN ${ }^{1}$ and BO LI ${ }^{1}$ \\ ${ }^{1}$ Department of Epidemiology and Statistics, School of Public Health, Jilin University, Changchun, Jilin 130021; \\ ${ }^{2}$ Department of Interventional Treatment, Cancer Hospital and Institution, \\ Tianjin Medical University, Tianjin 300060, P.R. China
}

Received January 26, 2015; Accepted March 30, 2016

DOI: $10.3892 / \mathrm{etm} .2016 .3251$

\begin{abstract}
Previous epidemiological studies have presented conflicting results regarding associations between circulating adiponectin (APN) levels and the risk of endometrial cancer. Thus a meta-analysis was performed to investigate the association between these factors. Multiple electronic sources, including PubMed, SpringerLink and Google Scholar databases were searched to identify relevant studies for the present meta-analysis. All of the selected studies examined the correlation between circulating APN levels and endometrial cancer. The standardized mean difference (SMD) and 95\% confidence intervals (CIs) were estimated and pooled using meta-analysis methods. Overall, 18 case-control studies met the inclusion criteria. A total of 5,692 participants and 2,337 cases of endometrial cancer were included in this meta-analysis. The SMD of the pooled analysis (95\% CI) were $-1.96(-2.60,-1.31)$, $\mathrm{P}=0.000$. When the cancer grades were compared, the APN values were not significantly different between the grades of endometrial cancer [G1 vs. G3, 1.02 (-0.68, 2.72), P>0.05; G1 vs. G2, 0.34 (-0.86, 1.54), P>0.05]. However, there was a significant association between high APN levels and postmenopausal endometrial cancer cases with an SMD (95\% CI) of $-2.27(-4.36,-0.18)$ and $\mathrm{P}<0.05$, however, no association was observed in premenopausal endometrial cancer cases with an SMD $(95 \% \mathrm{CI})$ of $-1.52(-3.49,0.45)$ and $\mathrm{P}>0.05$. The low circulating APN level increases the risk of endometrial cancer, whereas the high APN level decreases this risk in postmenopausal women. Circulating APN as simple biomarkers may be a promising tool for the prevention, early diagnosis and disease monitoring of endometrial cancer.
\end{abstract}

Correspondence to: Professor Bo Li, Department of Epidemiology and Statistics, School of Public Health, Jilin University, 1163 Xinmin Street, Changchun, Jilin 130021, P.R. China E-mail: li_bo@jlu.edu.cn

*Contributed equally

Key words: endometrial cancer, adiponectin, meta-analysis

\section{Introduction}

Endometrial cancer is the most common gynecological malignancy and the fourth most common type of cancer in women (1-3), following breast, lung and colorectal cancer. The incidence and mortality rate of endometrial cancer is increasing in developing and developed countries (3-6), although the rates of several other types of cancer have plateaued or decreased in the last decade (5). Over the past decades, much has been discovered about the genetics and lifestyle and environmental factors that affect endometrial cancer $(7,8)$. The most significant risk factors for endometrial cancer and its precursor, endometrial hyperplasia, are unopposed estrogen, a sedentary lifestyle and obesity $(5,9)$. Obesity is a major public health issue and continues to increase worldwide. By 2030, the rate of obesity is anticipated to reach $\sim 50 \%$ of the population of the US and $30 \%$ in Europe (10). Obesity not only significantly increases the risk of insulin resistance and type- 2 diabetes but it has also been identified as a risk factor for several types of cancer, such as endometrial cancer (10). Previous studies demonstrated that obesity is one of the strongest risk factors that exists for numerous types of cancer, including breast, colorectal, kidney, endometrial, ovarian, pancreatic and liver cancer (5,11-13). In addition, endometrial cancer was the first type to be shown to be associated with obesity (5).

Adipose tissue functions as an energy storage and also as a highly active and large source of endocrine and metabolic activity $(7,10)$. Adipokines are produced by adipose tissue and include leptin, resistin, adiponectin (APN), serpin, plasminogen activator inhibitor-1, retinol binding protein 4 and other numerous cytokines including tumor necrosis factor- $\alpha$ and interleukin receptor agonists (IL-1, IL-6, IL-8 and IL-10) $(14,15)$. APN is the most abundant adipokine that is secreted mainly from visceral adipose tissue and only from mature adipocytes. It is an endogenous insulin-sensitizing hormone, and is linked to obesity, metabolic syndrome, insulin resistance, type- 2 diabetes and inflammation in addition to several other types of cancer. APN has anti-diabetic, anti-angiogenic, anti-inflammatory and anti-atherogenic properties (10,14-16), which are known to inhibit cell proliferation, promote apoptosis and angiogenesis in tumor biology (17). Furthermore, APN inhibits carcinogenesis directly and indirectly by regulating hormone and cytokine levels. 


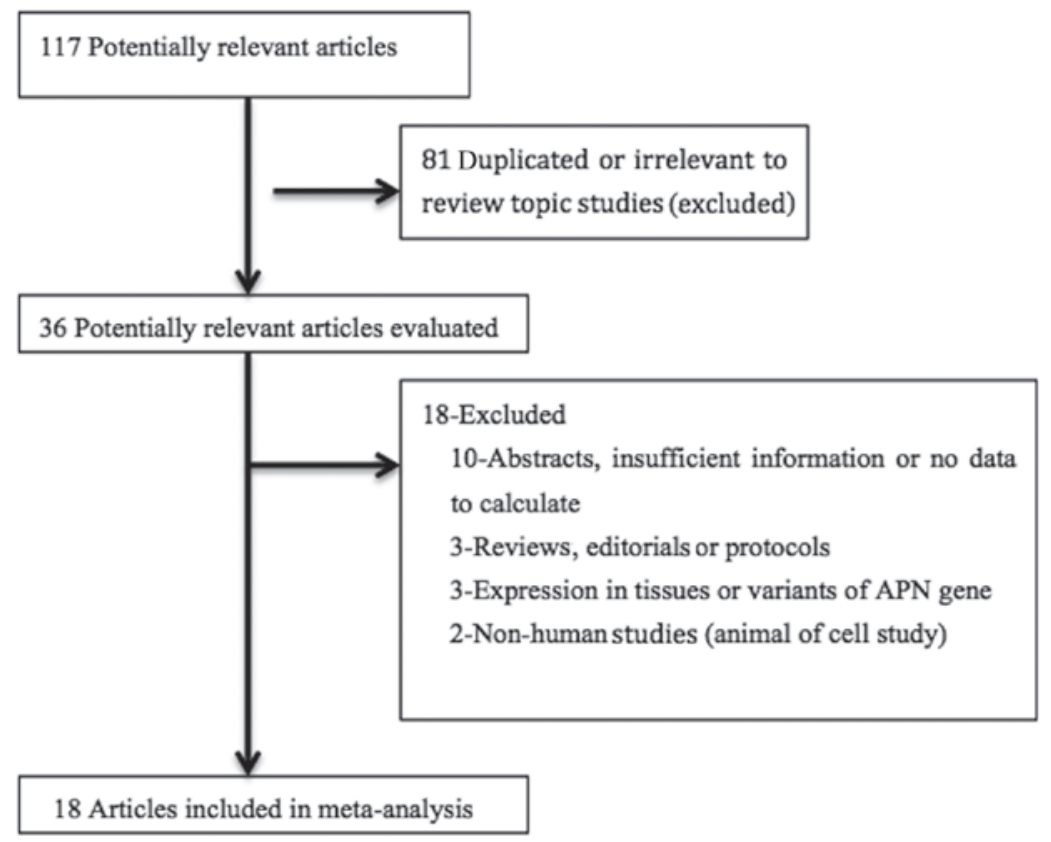

Figure 1. Flow chart of study identification.

Circulating APN may potentially be a protective factor for endometrial cancer. Retrospective and prospective studies have investigated the association of circulating APN levels with the risk of endometrial cancer. Although the majority of the studies demonstrated a negative association, several studies demonstrated that there is no association between the two. Therefore, the association between the two remains to be clarified. Nevertheless, the incidence of endometrial cancer has risen in each year over the past few years and the major problem facing treatment is its late diagnosis. By the time that it gets diagnosed, the vast majority of patients have advanced to the late stages of cancer, and the outcome of clinical treatment is poor. Therefore, is an interest amongst cancer researchers to identify convenient and effective molecular markers and methods in order to diagnose cancer at the early stages. Blood is the most readily available sample and it is an ideal specimen for identifying molecular markers of cancer. Circulating APN may be a novel molecular marker or anti tumor factor that may provide a feasible method for early detection and prevention of endometrial cancer which may be important in its diagnosis and therapy. To the best of our knowledge, there have been a few systematic reviews conducted to evaluate whether low APN levels increase the risk of endometrial cancer. Therefore, this meta-analysis was performed to summarize all of the data available and to provide a quantitative assessment of the risk.

\section{Patients and methods}

Characteristics of included studies. A total of 18 studies were included in the final analysis (18-35). The detailed steps of the search results are provided in Fig. 1. These 18 studies were published between 2003 and 2014, and included 2,337 women with endometrial cancer and 3,355 controls. Out of those 18 studies, 11 studies were conducted in Asia (China and Japan), 4 in North America (USA and Canada), and 3 in
Europe (Greece, Poland and Romania). The quality score of the studies ranged from 5 to 8 stars (Newcastle-Ottawa Scale) (36). There were only 7 studies providing the cancer grade data $(22-24,28,30,32,34)$. The main characteristics of the included studies are summarized in Tables I and II.

Search strategy. The US National Library of Medicine's PubMed database (http://www.ncbi.nlm.nih.gov/pubmed/) was searched using the terms 'endometrial cancer' 'endometrial carcinoma' 'adiponectin' 'Adipo' and 'APN' to assess the possibility of conducting the present study. A comprehensive literature search was conducted in PubMed, SpringerLink (http://link.springer.com/) and Google Scholar (http://scholar. google.com.hk) up to June 2014 using the terms 'adiponectin' or 'Adipo' or 'APN' and 'endometrial cancer' or 'endometrial carcinoma'. The search was limited to English and Chinese. Additional studies were also identified by manually searching references of original studies and by reviewing articles on the topic. Eligible studies in the current meta-analysis were those of case-control design, which reported the correlation between circulating APN levels and endometrial cancer. The present study was performed in accordance to the MOOSE guidelines for meta-analysis (37).

Data extraction. Data were independently extracted by two researchers. For each eligible study, the following information was recorded: The first author, year of publication, ethnicity, study size and number of cases, APN measurement method, total APN mean and standard deviation (38), menopausal status, control source and health status and language of publication. For one study (18) that provided the outcomes in medians and interquartile ranges, these were converted to the means and standard deviations (39). Any missing data from studies was obtained by communicating with the authors. The quality of each study was assessed by Newcastle-Ottawa Scaling (36). 


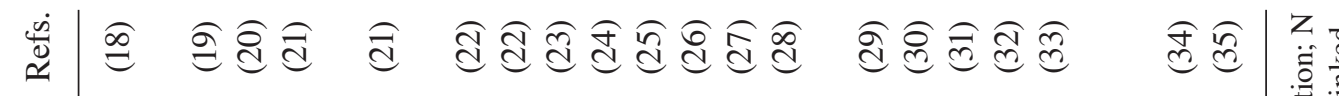

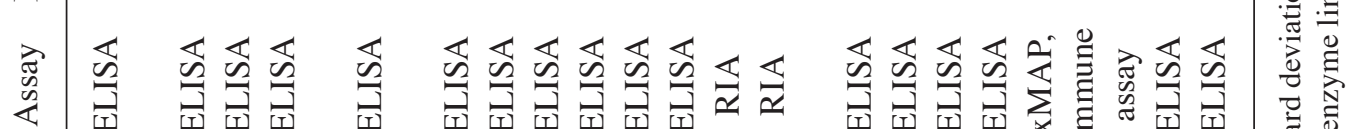

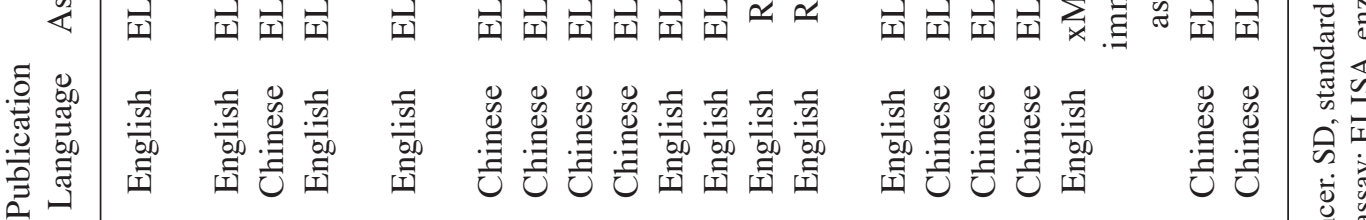

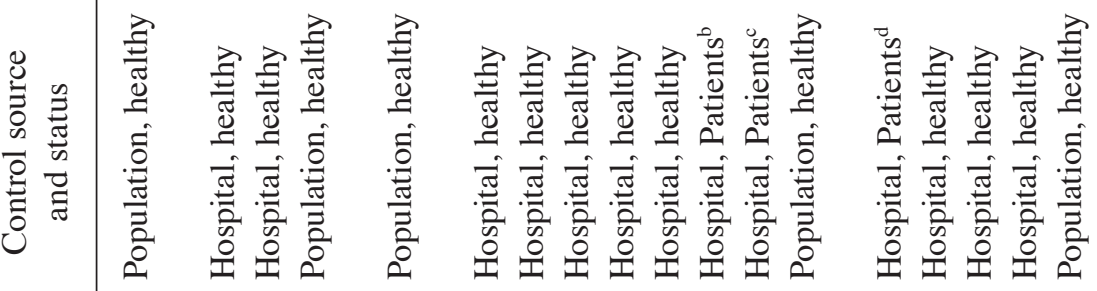

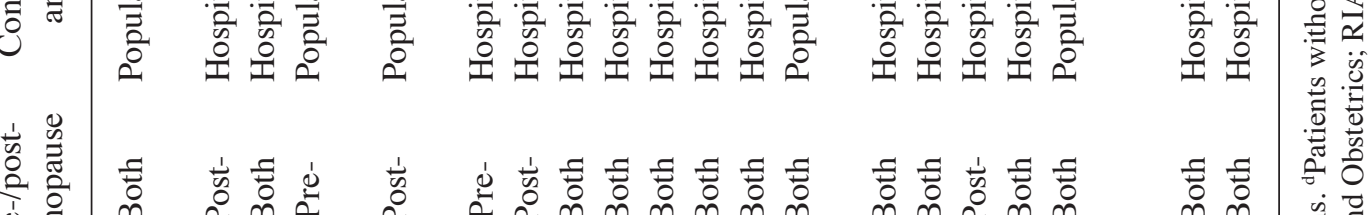

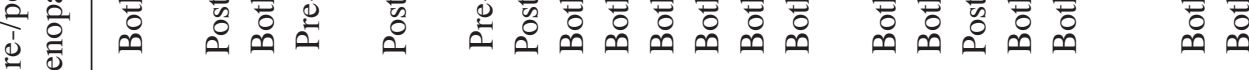

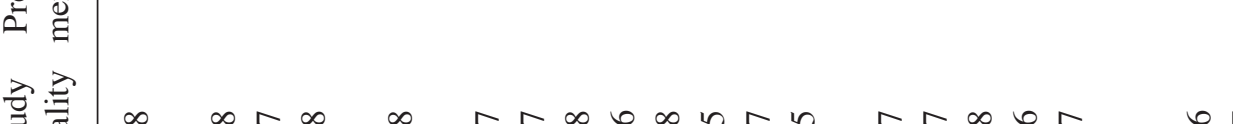

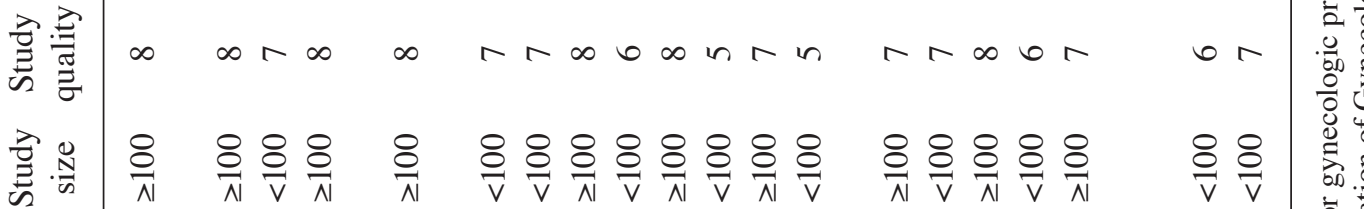

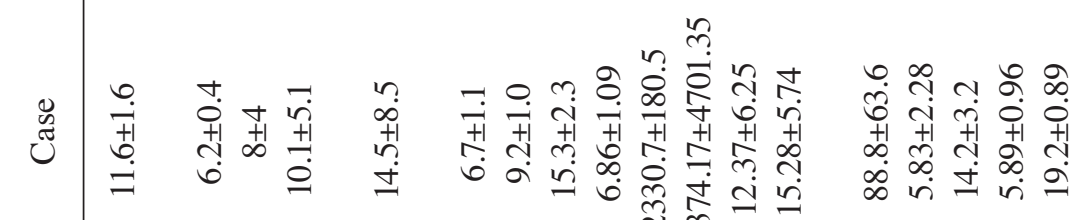

in

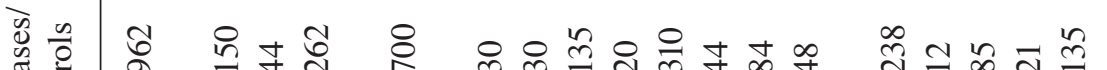

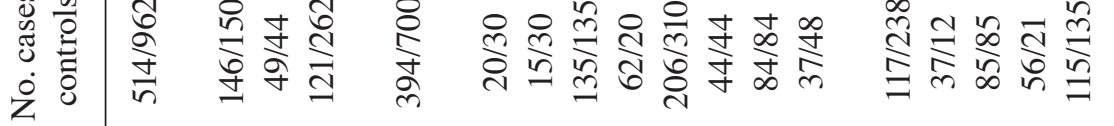
营

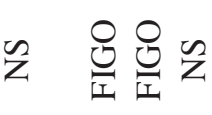

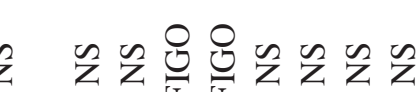

용 \&

在焉

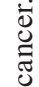

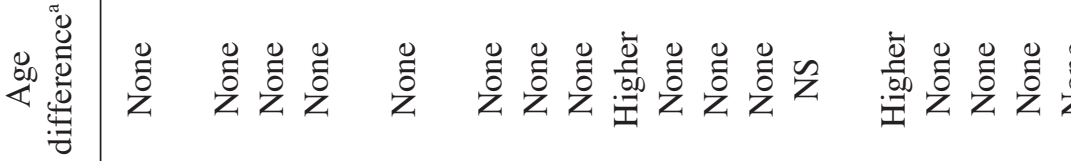

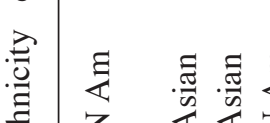


Table II. Characteristics of eligible studies in this meta-analysis depending on grade.

\begin{tabular}{|c|c|c|c|c|c|c|c|}
\hline \multirow[b]{2}{*}{ First author, year } & \multicolumn{3}{|c|}{ No. cases } & \multicolumn{3}{|c|}{ Adiponectin level (mean \pm standard deviation) } & \multirow[b]{2}{*}{ Refs. } \\
\hline & G1 & $\mathrm{G} 2$ & G3 & G1 & G2 & G3 & \\
\hline Fu, 2012 & 6 & 24 & 5 & $6.1 \pm 0.7$ & $7.9 \pm 1.4$ & $9.6 \pm 1.2$ & $(22)$ \\
\hline Wang, 2013 & 74 & 42 & 19 & $13.2 \pm 1.2$ & $9.6 \pm 1.4$ & $6.2 \pm 0.9$ & (23) \\
\hline $\mathrm{Li}, 2011$ & 17 & 62 & 27 & $6.37 \pm 0.61$ & $6.89 \pm 0.62$ & $6.45 \pm 0.59$ & (24) \\
\hline Rzepka-Górska, 2008 & 12 & 13 & 8 & $19.04 \pm 9.43$ & $13.48 \pm 5.95$ & $12.86 \pm 1.25$ & (28) \\
\hline Tan, 2012 & 12 & 37 & 10 & $7.98 \pm 2.18$ & $5.49 \pm 1.48$ & $3.74 \pm 0.65$ & (30) \\
\hline $\mathrm{Xu}, 2014$ & 27 & 19 & 10 & $6.06 \pm 0.72$ & $6.83 \pm 0.75$ & $6.22 \pm 0.94$ & (32) \\
\hline Zhang, 2010 & 40 & 17 & 7 & $9.31 \pm 4.65$ & $7.11 \pm 2.18$ & $4.47 \pm 1.35$ & (34) \\
\hline
\end{tabular}

Statistical analysis. The standardized mean differences (SMDs) of the total circulating APN were calculated for all of the eligible studies in the meta-analysis, as they were measured in different units across studies. A fixed effects model was used to pool the data when the P-value of the Q-test was $\geq 0.05$; otherwise a random effects model was selected (40). The $Q$ and $I^{2}$ statistics were used in order to evaluate the heterogeneity amongst the results of the studies. When $I^{2}>50 \%$, the heterogeneity was considered significant (41). A sensitivity analysis was conducted by exclusion of the studies; subgroup analysis according to study size, study quality, ethnicity, menopausal status, control source, language of publication and laboratory assay. Cumulative meta-analyses were performed in order to observe the change of the association with time and sample size (42). Furthermore, a funnel plot and Egger's test was used to assess the publication bias, and $\mathrm{P}<0.05$ was considered to indicate a statistically significant difference $(43,44)$. All the analyses were performed using Stata software version 11.0 (StataCorp LP, College Station, TX, USA).

\section{Results}

Main analysis. The results of the meta-analysis of the association between circulating APN levels and endometrial cancer included 20 data sets from 18 studies (Fig. 2). It was identified that women with endometrial cancer had significantly lower APN levels in the pooled analysis using a random-effects model [SMD (95\% CI), $-1.96(-2.60,-1.31)$; $\mathrm{P}<0.001$ ], yet with significant heterogeneity among studies $\left(I^{2}>50 \%\right)$. Egger's test for publication bias did not provide evidence of significant effect $(\mathrm{P}>0.05)$. There were 7 studies providing cancer grade. On comparison of cancer grades, APN values were not statistically different between the different grades of endometrial cancer [G1 vs. G3, $1.02(-0.68,2.72), \mathrm{P}>0.05 ; \mathrm{G} 1$ vs. G2, 0.34 $(-0.86,1.54), \mathrm{P}>0.05]$.

Subgroup/sensitivity analysis. Sensitivity analysis was performed by deleting one study at a time, and the pooled SMD for the remaining studies was calculated in order to check the influence of the removed data. The results revealed that 5 studies were the key contributors to the heterogeneity among studies (Fig. 3) $(19,23,24,32,33)$. Although heterogeneity existed after excluding these studies, the results revealed no significant alteration with an SMD (95\% CI) of -1.288
$(-1.655,-0.922)$ and $\mathrm{P}<0.001$. To identify potential sources of heterogeneity between studies, subgroup analysis was also performed by study size and quality, ethnicity, menopausal status, language of publication, control source and laboratory assay. The heterogeneity existed in subgroup analysis, and the association between small-sized and low-quality studies was identified to be stronger than in large-sized and high-quality studies. The present study suggested that the difference in study size and quality of the cases and the controls was due to the heterogeneity amongst the studies. The total effect values in Asia, Europe and North America were -2.48 (-3.97, -0.99), $-0.52(-0.90,-0.14)$ and $-1.71(-2.60,-0.82)$, respectively. The total effect value of postmenopausal endometrial cancer patients was $-2.27(-4.36,-0.18)$, which revealed that the high APN level was associated with decreased endometrial cancer risk in postmenopausal women $(\mathrm{P}<0.05)$. However, there was no association in premenopausal endometrial cancer [-1.52 $(-3.49,0.45), P>0.05]$. Furthermore, the risk of low APN level to endometrial cancer did not differ substantially by laboratory assay, control sources and language of publication. These results are presented in Table III.

Cumulative meta-analysis. To further observe the change in the association between circulating APN levels and endometrial cancer, the cumulative meta-analysis was performed and sorted by year and total size (Figs. 4 and 5). Data analysis suggests that the risk of low APN levels to endometrial cancer was not observed all the time and requires further study in order to confirm this result. Nevertheless, patients with endometrial cancer were observed to have markedly lower APN values compared with the controls.

Estimation of publication bias. Begg's funnel plot and Egger's test were performed to assess the publication bias. The Funnel plot revealed a slight asymmetry in the distribution but Egger's regression test suggested no significant asymmetry of the Funnel plot $(\mathrm{P}=0.229)$.

\section{Discussion}

The majority of previous studies have suggested that there is an independent and negative association of circulating APN with endometrial cancer, however, a number of studies indicated no association $(29,45,46)$. Since the results of a single study may 


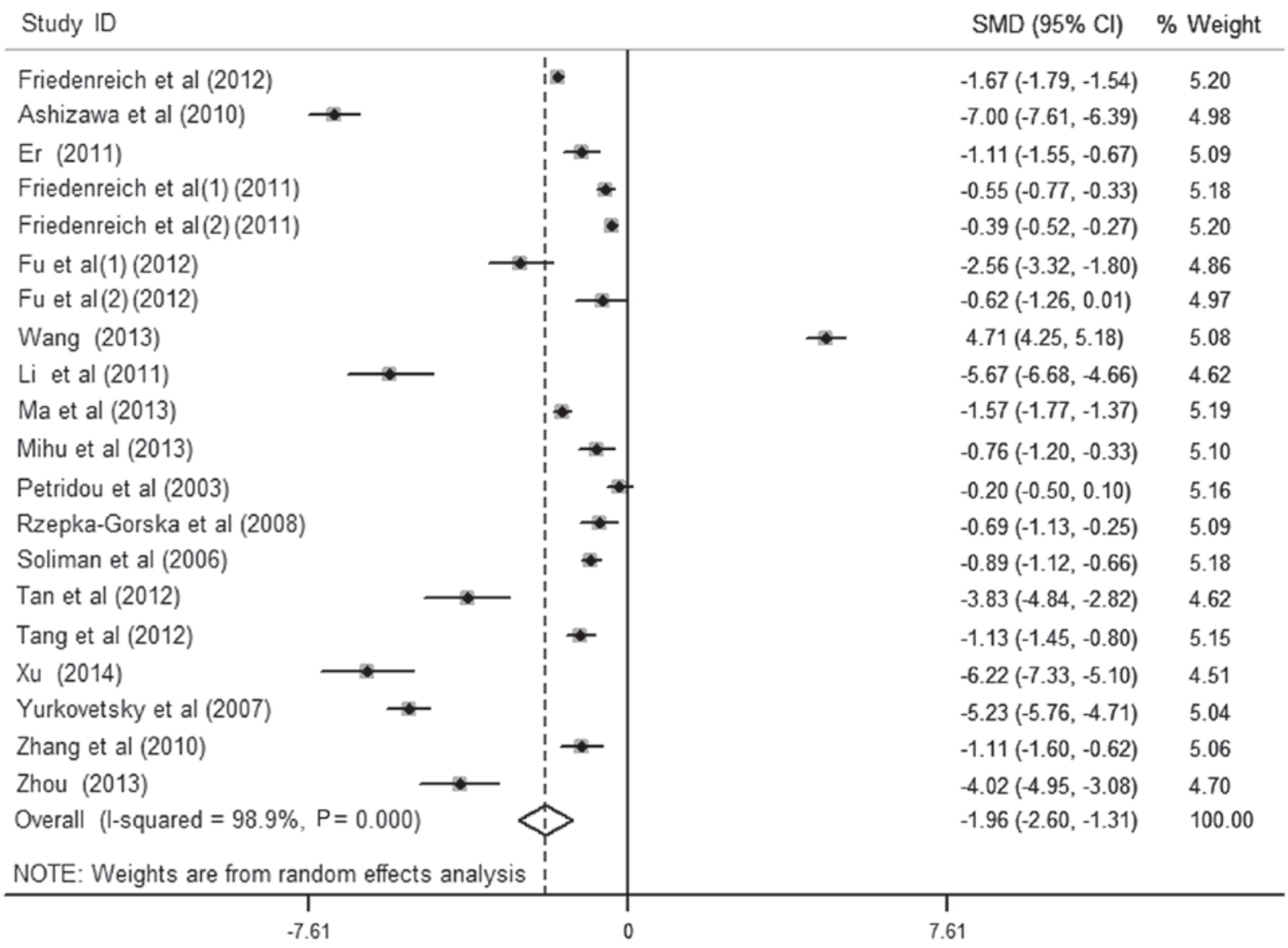

Figure 2. SMD adiponectin values in endometrial cancer and matched controls and pooled estimate (random effects). CI, confidence interval; SMD, standardized mean difference.

\begin{tabular}{|c|c|c|c|}
\hline Study ID & & $\operatorname{SMD}(95 \% \mathrm{Cl})$ & $\%$ Weight \\
\hline Friedenreich et al (2012) & & $-1.67(-1.79,-1.54)$ & 7.47 \\
\hline Er (2011) & & $-1.11(-1.55,-0.67)$ & 6.81 \\
\hline Friedenreich et al(1) (2011) & $\leftarrow$ & $-0.55(-0.77,-0.33)$ & 7.34 \\
\hline Friedenreich et al(2) (2011) & $*$ & $-0.39(-0.52 .-0.27)$ & 7.47 \\
\hline Fu et al(1) (2012) & & $-2.56(-3.32,-1.80)$ & 5.68 \\
\hline Fu et al(2) (2012) & & $-0.62(-1.26,0.01)$ & 6.15 \\
\hline Ma et al (2013) & & $-1.57(-1.77,-1.37)$ & 7.37 \\
\hline Mihu et al (2013) & $-\infty$ & $-0.76(-1.20,-0.33)$ & 6.82 \\
\hline Petridou et al (2003) & $\rightarrow$ & $-0.20(-0.50,0.10)$ & 7.17 \\
\hline Rzepka-Gorska et al (2008) & $\rightarrow-$ & $-0.69(-1.13,-0.25)$ & 6.79 \\
\hline Soliman et al (2006) & + & $-0.89(-1.12,-0.66)$ & 7.32 \\
\hline Tan et al (2012) & & $-3.83(-4.84,-2.82)$ & 4.79 \\
\hline Tang et al (2012) & & $-1.13(-1.45,-0.80)$ & 7.12 \\
\hline Zhang et al (2010) & & $-1.11(-1.60,-0.62)$ & 6.64 \\
\hline Zhou (2013) & & $-4.02(-4.95,-3.08)$ & 5.06 \\
\hline Overall (I-squared $=96.2 \%, P=0.000$ ) & & $-1.29(-1.66,-0.92)$ & 100.00 \\
\hline NOTE: Weights are from random effects analysis & & & \\
\hline $\begin{array}{c}1 \\
-4.95\end{array}$ & & 9 & \\
\hline
\end{tabular}

Figure 3. Effect of circulating adiponectin on endometrial cancer. CI, confidence interval; SMD, standardized mean difference. 
Table III. Stratified meta-analysis of circulating adiponectin levels and endometrial cancer.

\begin{tabular}{|c|c|c|c|c|c|c|}
\hline \multirow[b]{2}{*}{ Characteristic } & \multirow[b]{2}{*}{ Data points $(\mathrm{N})$} & \multicolumn{2}{|c|}{ Endometrial cancer $(\mathrm{N})$} & \multirow[b]{2}{*}{ Random effects } & \multirow[b]{2}{*}{ P-value } & \multirow[b]{2}{*}{$I^{2}(\%)$} \\
\hline & & Yes & No & & & \\
\hline All studies & 20 & 2337 & 3355 & $-1.96(-2.60,-1.31)$ & 0.000 & 98.9 \\
\hline \multicolumn{7}{|l|}{ Study size } \\
\hline$<100$ & 10 & 420 & 294 & $-2.58(-3.57,-1.60)$ & 0.000 & 96.0 \\
\hline$\geq 100$ & 10 & 1917 & 3061 & $-1.37(-2.28,-0.46)$ & 0.003 & 99.4 \\
\hline \multicolumn{7}{|l|}{ Study quality } \\
\hline Low (score <7) & 5 & 263 & 158 & $-2.81(-4.46,-1.16)$ & 0.001 & 97.5 \\
\hline High (score $\geq 7$ ) & 15 & 2074 & 3197 & $-1.70(-2.44,-0.96)$ & 0.000 & 99.1 \\
\hline \multicolumn{7}{|l|}{ Ethnicity } \\
\hline Asia & 12 & 911 & 882 & $-2.48(-3.97,-0.99)$ & 0.001 & 99.1 \\
\hline Europe & 3 & 165 & 176 & $-0.52(-0.90,-0.14)$ & 0.007 & 65.1 \\
\hline North America & 5 & 1261 & 2297 & $-1.71(-2.60,-0.82)$ & 0.000 & 99.2 \\
\hline \multicolumn{7}{|l|}{ Pre-/post-menopause } \\
\hline Both & 14 & 1556 & 2098 & $-1.95(-2.81,-1.08)$ & 0.000 & 98.9 \\
\hline Pre- & 2 & 141 & 292 & $-1.52(-3.49,0.45)$ & 0.131 & 95.9 \\
\hline Post- & 4 & 640 & 965 & $-2.27(-4.36,-0.18)$ & 0.033 & 99.3 \\
\hline \multicolumn{7}{|l|}{ Laboratory assay } \\
\hline ELISA & 17 & 2101 & 3088 & $-1.94(-2.62,-1.26)$ & 0.000 & 98.9 \\
\hline RIA & 2 & 121 & 132 & $-1.96(-2.60,-1.31)$ & 0.086 & 68.6 \\
\hline xMAP & 1 & 115 & 135 & $-5.23(-5.76,-4.71)$ & - & - \\
\hline \multicolumn{7}{|l|}{ Control source } \\
\hline Hospital & 15 & 1156 & 1248 & $-2.09(-3.12,-1.06)$ & 0.000 & 98.9 \\
\hline Population & 5 & 1181 & 2107 & $-1.68(-2.63,-0.72)$ & 0.001 & 99.2 \\
\hline \multicolumn{7}{|l|}{ Control status } \\
\hline Healthy & 17 & 2063 & 3077 & $-1.83(-2.48,-1.17)$ & 0.000 & 98.8 \\
\hline Patients without EC & 3 & 274 & 278 & $-2.65(-6.13,0.85)$ & 0.137 & 99.5 \\
\hline \multicolumn{7}{|l|}{ Publication language } \\
\hline English & 10 & 1778 & 2933 & $-1.86(-2.57,-1.14)$ & 0.000 & 99.0 \\
\hline Chinese & 10 & 559 & 422 & $-2.13(-3.91,-0.34)$ & 0.019 & 98.9 \\
\hline
\end{tabular}

Random effects data are presented as the standardized mean difference (95\% confidence interval). RIA, radioimmunoassay; EC, endometrial cancer.

be affected by numerous factors, the present study included 18 studies, which comprised a total of 4,186 participants and 1,823 cases, to further detect the risk of low APN in endometrial cancer by reducing the bias and by increasing the efficiency of the statistics. In the present study, significantly low APN levels were observed in women with endometrial cancer in the pooled analysis, although there was clear heterogeneity across the studies. When the cancer grades were compared, the risk of low APN to endometrial cancer was not significantly different between grades of endometrial cancer, which was not identical with previous studies $(22-24,28,30,32,34)$. In the subgroup and sensitivity analyses, there was significant heterogeneity among studies. The significant association existed in all of the pooled analysis, but not in premenopausal women, the radioimmunoassay or patients without EC from control sources. The risk was identified to be stronger in small-sized and low-quality studies than in large-sized and high-quality studies, suggesting that the effect of APN may be strongly affected by the small-sized and low-quality studies. A previous study indicated that high APN levels may decrease the risk of postmenopausal endometrial cancer (28). However, there was no significant difference in premenopausal women, which was in contrast with various other studies $(29,47,48)$. Since the female gender and hormone levels are recognized risk factors for endometrial cancer and the mechanism remains unclear, the risk of low APN levels on endometrial cancer of pre- and postmenopausal women is difficult to fully investigate. In the other subgroups, no significant differences were observed in the risk of low APN to endometrial cancer. In the cumulative meta-analysis that was undertaken in the present study, the risk of low APN to endometrial cancer changed with time and sample size. Therefore, further studies are required in order to identify the risk.

The mechanisms of action of APN in regards to endometrial cancer have not yet been fully elucidated. APN is a link 
Study ID

$\operatorname{SMD}(95 \% \mathrm{Cl})$

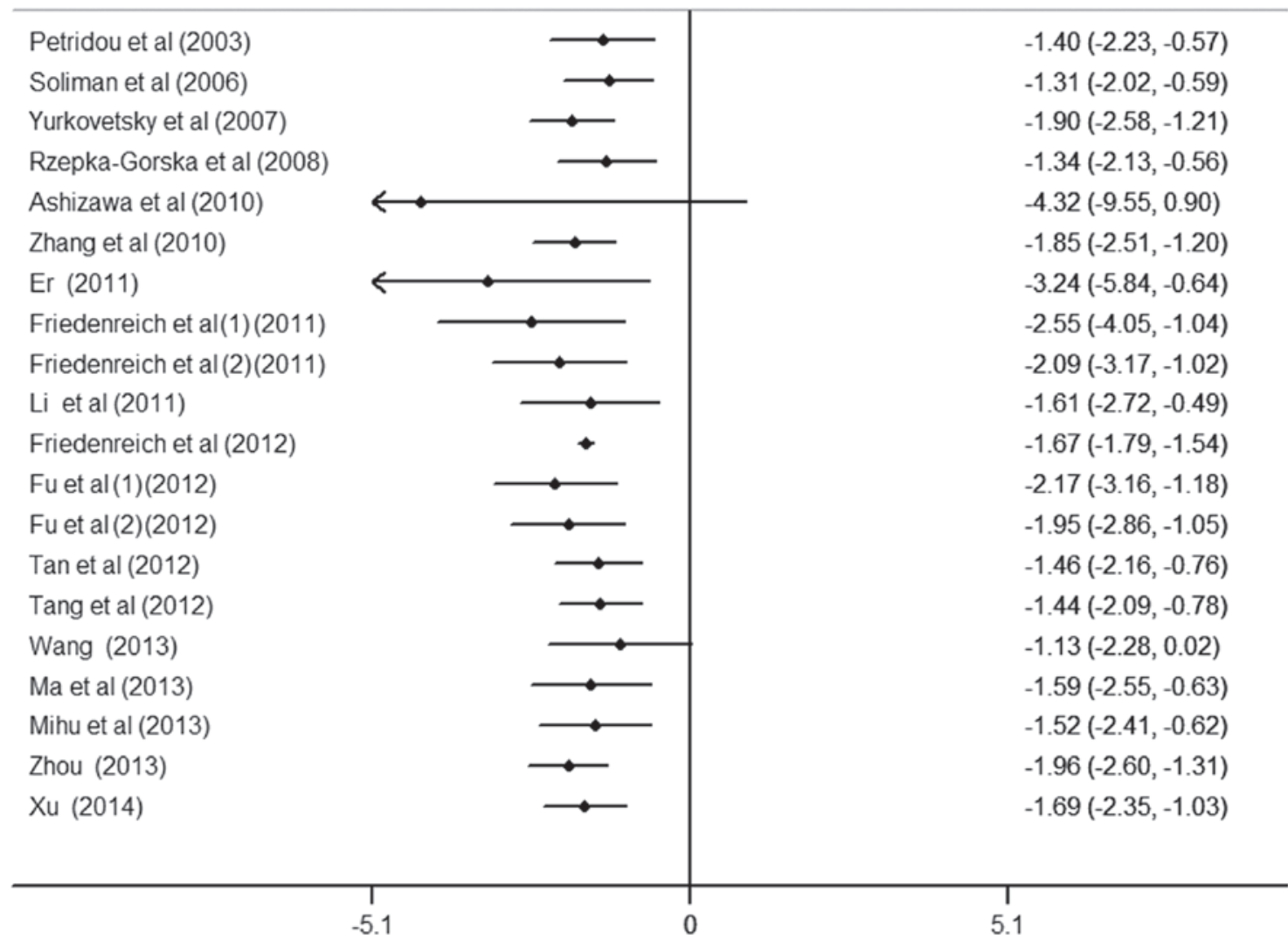

Figure 4. Cumulative analysis by year of circulating adiponectin on endometrial cancer. CI, confidence interval; SMD, standardized mean difference.

Study ID

Fu et al (2)(2012)

Tan et al (2012)

Fu et al(1)(2012)

Zhou (2013)

Xu (2014)

Li et al (2011)

Rzepka-Gorska et al (2008)

Mihu et al (2013)

Zhang et al (2010)

Er (2011)

Petridou et al (2003)

Tang et al (2012)

Yurkovetsky et al (2007)

Wang (2013)

Ashizawa et al (2010)

Soliman et al (2006)

Friedenreich et al(1)(2011)

Ma et al (2013)

Friedenreich et al (2)(2011)

Friedenreich et al (2012)
$\operatorname{SMD}(95 \% \mathrm{Cl})$

$-1.95(-2.86,-1.05)$

$-1.46(-2.16,-0.76)$

$-2.17(-3.16,-1.18)$

$-1.96(-2.60,-1.31)$

$-1.69(-2.35,-1.03)$

$-1.61(-2.72,-0.49)$

$-1.34(-2.13,-0.56)$

$-1.52(-2.41,-0.62)$

$-1.85(-2.51,-1.20)$

$-3.24(-5.84,-0.64)$

$-1.40(-2.23,-0.57)$

$-1.44(-2.09,-0.78)$

$-1.90(-2.58,-1.21)$

$-1.13(-2.28,0.02)$

$-4.32(-9.55,0.90)$

$-1.31(-2.02,-0.59)$

$-2.55(-4.05,-1.04)$

$-1.59(-2.55,-0.63)$

$-2.09(-3.17,-1.02)$

$-1.67(-1.79,-1.54)$ 
between obesity and obesity-associated malignancies, mainly on the basis of the finding that APN levels are lower in patients who suffer with these types of cancer. AdipoR1/AdipoR2 are the two different receptor isoforms of APN, which are expressed in fat and muscle tissues in humans. The expression levels of these receptors are regulated by insulin and peroxisome proliferator-activated receptors (PPAR) $-\alpha$ and $-\gamma$. It has been demonstrated that the receptors have lower expression levels in subcutaneous and visceral adipose tissues than in skeletal muscle. Furthermore, previous studies confirmed that there was a strong, inverse and consistent association between circulating APN and obesity, insulin resistance and inflammatory markers, including C-reactive protein and fibrinogen. Thus, several case-control studies demonstrated that APN receptors, particularly AdipoR1, are upregulated in malignancies such as endometrial cancer. Furthermore, there is a possibility that APN affects cancer cell proliferation and tumor formation and progression directly by receptor-mediated stimulation of signaling pathways and indirectly by moderating insulin sensitivity. APN binding to its receptors (AdipoR1/AdipoR2) may exert direct effects on these malignancy signaling pathways, mainly $5^{\prime}$ adenosine monophosphate-activated protein kinase (AMPK), but also PPAR- $\alpha$, mitogen-activated protein kinase and nuclear factor (NF)-кB. AMPK activation regulates cell proliferation, decreases the expression of transcriptional regulators and positively regulates important proteins associated with controlling cell cycle arrest and apoptosis. It is suggested that APN may affect tumor cells by directly inhibiting pro-angiogenic factors, including basic fibroblast growth factor and interleukin- 8 produced by tumors, or platelet-derived growth factor BB produced by endothelial cells. APN also exerts an indirect action by insulin-sensitizing, anti-inflammatory and anti-angiogenic effects. Hyperinsulinemia, obesity and physical inactivity result in higher concentrations of circulating sex hormone-binding globulin and insulin-like growth factor 1, which leads to increased cell proliferation, decreased apoptosis and increased inflammation. In vitro studies to date reveal that APN decreases cell viability and proliferation in endometrial cancer. Although these results are promising, further studies are required in animals and humans to elucidate the effects of circulating APN and its association with endometrial cancer.

To the best of our knowledge, the present study was the first to perform a meta-analysis that adds to the current understanding of the association between circulating APN levels and endometrial cancer. The present study included a large number of cases and controls that significantly increased its statistical power. However, several limitations in the study should be addressed. The study is based on observational studies (case-control) and is particularly vulnerable to potential biases (information or selection bias) and bias in the original studies. The significant heterogeneity observed across the results of the study reduces the reliability of the observations, suggesting different outcomes across studies with different patients, controls, methods and measurements. All eligible studies were published in English and Chinese and a number of relevant studies in other languages were not included. Additionally, the conversion of non-normally distributed statistics maybe have been less accurate than if individual data had been available. Finally, the sample sizes for certain subgroup analyses were small. Therefore, the conclusions drawn from the results of this meta-analysis should be regarded cautiously.

In summary, the present study indicated that low circulating APN levels may increase the risk of endometrial cancer, although there was a significant heterogeneity across the studies, and that the high APN level may decrease the risk of endometrial cancer in postmenopausal women. As APN is theorized to affect cell proliferation, tumor formation and progression of endometrial cancer, it may be a promising tool in endometrial cancer prevention, diagnosis and therapy as a simple biomarker. However, further studies are required in order to elucidate the mechanisms of APN.

\section{Acknowledgements}

The authors are very grateful to Dr Petridou for providing the additional data requested. This present research was supported by the Graduate Innovation Fund of Jilin University (grant no. 2014113).

\section{References}

1. Burzawa JK, Schmeler KM, Soliman PT, Meyer LA, Bevers MW, Pustilnik TL, Anderson ML, Ramondetta LM, Tortolero-Luna G, Urbauer DL, et al: Prospective evaluation of insulin resistance among endometrial cancer patients. Am J Obstet Gynecol 204: 355.e1-355.e7, 2011.

2. Lucenteforte E, Bosetti C, Talamini R, Montella M, Zucchetto A, Pelucchi C, Franceschi S, Negri E, Levi F and La Vecchia C: Diabetes and endometrial cancer: Effect modification by body weight, physical activity and hypertension. Br J Cancer 97: 995-998, 2007.

3. Yamauchi N, Takazawa Y, Maeda D, Hibiya T, Tanaka M, Iwabu M, Okada-Iwabu M, Yamauchi T, Kadowaki T and Fukayama M: Expression levels of adiponectin receptors are decreased in human endometrial adenocarcinoma tissues. Int J Gynecol Pathol 31: 352-357, 2012.

4. Yin XH, Jia HY, Xue XR, Yang SZ and Wang ZQ: Clinical analysis of endometrial cancer patients with obesity, diabetes and hypertension. Int J Clin Exp Med 7: 736-743, 2014.

5. Fader AN, Arriba LN, Frasure HE and von Gruenigen VE: Endometrial cancer and obesity: Epidemiology, biomarker, prevention and survivorship. Gynecol Oncol 114: 121-127, 2009.

6. Linkov F, Edwards R, Balk J, Yurkovetsky Z, Stadterman B, Lokshin A and Taioli E: Endometrial hyperplasia, endometrial cancer and prevention: Gaps in existing research of modifiable risk factors. Eur J Cancer 44: 1632-1644, 2008.

7. Xu XT, Xu Q, Tong JL, Zhu MM, Huang ML, Ran ZH and Xiao SD: Meta-analysis: Circulating adiponectin levels and risk of colorectal cancer and adenoma. J Dig Dis 12: 234-244, 2011.

8. Cust AE, Armstrong BK, Friedenreich CM, Slimani N and Bauman A: Physical activity and endometrial cancer risk: A review of the current evidence, biologic mechanisms and the quality of physical activity assessment methods. Cancer Causes Control 18: 243-258, 2007.

9. Schouten LJ,Goldbohm RA and van den Brandt PA: Anthropometry, physical activity, and endometrial cancer risk: Results from the Netherlands Cohort Study. J Natl Cancer Inst 96: 1635-1638, 2004.

10. Barb D, Pazaitou-Panayiotou K and Mantzoros CS: Adiponectin: A link between obesity and cancer. Expert Opin Investig Drugs 15: 917-931, 2006.

11. Calle EE and Kaaks R: Overweight, obesity and cancer: Epidemiological evidence and proposed mechanisms. Nat Rev Cancer 4: 579-591, 2004.

12. Joshi RK and Lee SA: Obesity related adipokines and colorectal cancer: A review and meta-analysis. Asian Pac J Cancer Prev 15: 397-405, 2014.

13. Renehan AG, Roberts DL and Dive C: Obesity and cancer: Pathophysiological and biological mechanisms. Arch Physiol Biochem 114: 71-83, 2008.

14. Dalamaga M, Diakopoulos KN and Mantzoros CS: The role of adiponectin in cancer: A review of current evidence. Endocr Rev 33: 547-594, 2012. 
15. Kelesidis I, Kelesidis T and Mantzoros CS: Adiponectin and cancer: A systematic review. Br J Cancer 94: 1221-1225, 2006.

16. Housa D, Housová J, VernerováZ and Haluzík M: Adipocytokines and Cancer. Physiol Res 55: 233-244, 2006.

17. Roberts DL, Dive C and Renehan AG: Biological mechanisms linking obesity and cancer risk: New perspectives. Annu Rey Med 61: 301-316, 2010.

18. Friedenreich CM,Langley AR,Speidel TP,Lau DC, Courneya KS Csizmadi I, Magliocco AM, Yasui Y and Cook LS: Case-control study of markers of insulin resistance and endometrial cancer risk. Endocr Relat Cancer 19: 785-792, 2012.

19. Ashizawa N, Yahata T, Quan J, Adachi S, Yoshihara K and Tanaka K: Serum leptin-adiponectin ratio and endometrial cancer risk in postmenopausal female subjects. Gynecol Oncol 119: 65-69, 2010

20. Er LX and Liu FQ: Roles of VEGF, adiponectin and EGFR in endometrial adenocarcinoma. Yi Xue Zong Shu 17: 1417-1418, 2011 (In Chinese).

21. Friedenreich CM, Biel RK, Lau DC, Csizmadi I, Courneya KS Magliocco AM, Yasui Y and Cook LS: Case-control study of the metabolic syndrome and metabolic risk factors for endometrial cancer. Cancer Epidemiol Biomarkers Prev 20: 2384-2395, 2011

22. Fu JR, Lu LN, Yu LP, Wang DY and Ding KS: Serum changes of adiponectin, insulin resistance and their correlation in endometrial cancer patients. Zhonghua Fu Chan Ke Za Zhi 47: 672-675, 2012 (In Chinese).

23. Wang G: Analysis of relationship between serum adiponectin level and insulin resisitance index in endomerial carcinoma. Shi Yong Ai Zheng Za Zhi She 28: 599-601, 2013 (In Chinese).

24. Li SP, Huang XH and Zhang AL: Expression of serum adiponectin in patients with endometrial cancer and its clinical significance. J Clin Res 28: 1252-1253, 2011.

25. Ma Y, Liu Z, Zhang Y and Lu B: Serum leptin, adiponectin and endometrial cancer risk in Chinese women. J Gynecol Oncol 24: 336-341, 2013

26. Mihu D, Ciortea R and Mihu CM: Abdominal adiposity through adipocyte secretion products, a risk factor for endometrial cancer. Gynecol Endocrinol 29: 448-451, 2013.

27. Petridou E, Mantzoros C, Dessypris N, Koukoulomatis P, Addy C, Voulgaris Z, Chrousos G and Trichopoulos D: Plasma adiponectin concentrations in relation to endometrial cancer: A case-control study in Greece. J Clin Endocrinol Metab 88 993-997, 2003

28. Rzepka-Górska I, Bedner R, Cymbaluk-Płoska A and Chudecka-Głaz A: Serum adiponectin in relation to endometrial cancer and endometrial hyperplasia with atypia in obese women. Eur J Gynaecol Oncol 29: 594-597, 2008.

29. Soliman PT, Wu D, Tortolero-Luna G, Schmeler KM, Slomovitz BM, Bray MS, Gershenson DM and Lu KH: Association between adiponectin, insulin resistance, and endometrial cancer. Cancer 106: 2376-2381, 2006.

30. Tan ZQ, Liu FX and Long D: Expression and clinical significance of serum adiponectin in patients with endometrial cancer. Guangdong Med J 33: 1757-1759, 2012.

31. Tang JM, Xiao Q and Fang Y: Study on the risk ofactors related to sign the comprehensive endometrial cancer and metabolism. Zhejiang Prev Med 24: 69-70, 2012.

32. Xu L: Relationship between the expression of adiponectin in serum and type-I Endometrial Carcinoma. Hua Xi Yi Xue 29: 736-737, 2014 (In Chinese).
33. Yurkovetsky Z, Ta'asan S, Skates S, Rand A, Lomakin A, Linkov F, Marrangoni A, Velikokhatnaya L, Winans M, Gorelik E, et al: Development of multimarker panel for early detection of endometrial cancer. High diagnostic power of prolactin. Gynecol Oncol 107: 58-65, 2007.

34. Zhang LZ, Wen K and Liu R: Change and significance of serum adiponectin level in patients with endometrial carcinoma. Shangdong Med 50: 4-5, 2010.

35. Zhou MN: Research on expression of adiponectin in serum of the patients with endometrial cancer and its clinical significance. Zhong Guo Wei Sheng Chan Ye Za Zhi She 10: 156-158, 2013 (In Chinese).

36. Wells GA, Shea B, O'connell D, et al: The Newcastle-Ottawa Scale (NOS) for assessing the quality of nonrandomised studies in meta-analyses. www.ohri.ca/programs/clinical_epidemiology/oxford.asp ACCESS DATE month, day, year.

37. Stroup DF, Berlin JA, Morton SC, Olkin I, Williamson GD, Rennie D, Moher D, Becker BJ, Sipe TA and Thacker SB: Meta-analysis of observational studies in epidemiology: A proposal for reporting. Meta-analysis Of Observational Studies in Epidemiology (MOOSE) group. JAMA 283: 2008-2012, 2000.

38. Pinthus JH, Kleinmann N, Tisdale B, Chatterjee S, Lu JP, Gillis A, Hamlet T, Singh G, Farrokhyar F and Kapoor A: Lower plasma adiponectin levels are associated with larger tumor size and metastasis in clear-cell carcinoma of the kidney. Eur Urol 54: 866-873, 2008

39. Hozo SP, Djulbegovic B and Hozo I: Estimating the mean and variance from the median, range, and the size of a sample. BMC Med Res Methodol 5: 13, 2005.

40. DerSimonian R: Meta-analysis in the design and monitoring of clinical trials. Stat Med 15: 1237-1252, 1996.

41. Higgins JP and Thompson SG: Quantifying heterogeneity in a meta-analysis. Stat Med 21: 1539-1558, 2002.

42. Leimu R and Koricheva J: Cumulative meta-analysis: A new tool for detection of temporal trends and publication bias in ecology. Proc Biol Sci 271: 1961-1966, 2004

43. Begg CB and Mazumdar M: Operating characteristics of a rank correlation test for publication bias. Biometrics 50: 1088-1101, 1994.

44. Egger M, Davey Smith G, Schneider M and Minder C: Bias in meta-analysis detected by a simple, graphical test. BMJ 315: 629-634, 1997

45. Dallal CM, Brinton LA, Bauer DC, Buist DS, Cauley JA, Hue TF, Lacroix A, Tice JA, Chia VM, Falk R, et al; B FIT Research Group: Obesity-related hormones and endometrial cancer among postmenopausal women: A nested case-control study within the B FIT cohort. Endocr Relat Cancer 20: 151-160, 2013.

46. Modesitt SC, Geffel DL, Via J and L Weltman A: Morbidly obese women with and without endometrial cancer: Are there differences in measured physical fitness, body composition, or hormones? Gynecol Oncol 124: 431-436, 2012.

47. Cust AE, Kaaks R, Friedenreich C, Bonnet F, Laville M, Lukanova A, Rinaldi S, Dossus L, Slimani N, Lundin E, et al: Plasma adiponectin levels and endometrial cancer risk in pre- and postmenopausal women. J Clin Endocrinol Metab 92: 255-263, 2007.

48. Dal Maso L, Augustin LS, Karalis A, Talamini R, Franceschi S, Trichopoulos D, Mantzoros CS and La Vecchia C: Circulating adiponectin and endometrial cancer risk. J Clin Endocrinol Metab 89: 1160-1163, 2004. 